\title{
New Results from the first 5 years of CALET observations on the International Space Station
}

Pier Simone Marrocchesi ${ }^{a, b, *}$ on behalf of the CALET Collaboration

(a complete list of authors can be found at the end of the proceedings)

${ }^{a}$ Department of Physical Sciences, Earth and Environment, University of Siena, via Roma 56, 53100 Siena, Italy

${ }^{b}$ INFN Sezione di Pisa, Polo Fibonacci, Largo B. Pontecorvo 3, 56127 Pisa, Italy

E-mail: marrocchesi@unisi.it

The CALorimetric Electron Telescope (CALET), developed and operated by Japan in collaboration with Italy and the United States, is a high-energy astroparticle physics experiment installed on the International Space Station (ISS). Its mission goals include investigating the possible presence of nearby sources of high-energy electrons, performing direct measurements of observables sensitive to the details of the acceleration and propagation of galactic particles, and detecting potential dark matter signatures. CALET measures cosmic-ray electron+positron flux up to $20 \mathrm{TeV}$, gamma rays up to $10 \mathrm{TeV}$, and nuclei up to $1,000 \mathrm{TeV}$. Charge measurements cover from $\mathrm{Z}=1$ to 40 allowing to study the more abundant elements and to extend the range of long-term observations above iron. CALET is collecting science data on the International Space Station since October 2015 with excellent and continuous performance with no major interruptions. Approximately 20 million triggered events per month are recorded with energies $>10 \mathrm{GeV}$. Here, we present the highlights of CALET observations carried out during the first 5.5 years of operation, including the electron+positron energy spectrum, the spectra of protons and other nuclei, gamma-ray observations, as well as the characterization of on-orbit performance. Some results on the electromagnetic counterpart search for LIGO/Virgo gravitational wave events and the observations of solar modulation and gamma-ray bursts are also included.

$37^{\text {th }}$ International Cosmic Ray Conference (ICRC 2021)

July 12th - 23rd, 2021

Online - Berlin, Germany

\footnotetext{
${ }^{*}$ Presenter
} 


\section{Introduction}

CALET is a space experiment aboard the ISS, designed for long-term observations of charged and neutral cosmic radiation. The instrument is managed by an international collaboration led by the Japanese Space Agency (JAXA) with the participation of the Italian Space Agency (ASI) and NASA. It was launched on August 19, 2015 with the Japanese carrier H-IIB, delivered to the ISS by the HTV-5 Transfer Vehicle, and installed on the Japanese Experiment Module Exposure Facility (JEM-EF).

The science program of CALET addresses several outstanding questions of high-energy astroparticle physics including the origin of cosmic rays (CR), the possible presence of nearby astrophysical CR sources, the acceleration and propagation of primary and secondary elements in the galaxy, and the nature of dark matter. The design of CALET is optimised for high precision measurements of the electron+positron spectrum with an accurate scan of the energy interval already covered by previous experiments and its extension to the region above $1 \mathrm{TeV}$. Given the high energy resolution of CALET for electrons, a detailed study of the spectral shape might reveal the presence of nearby sources of acceleration as well as possible indirect signatures of dark matter $[1,2]$. With its capability of identifying cosmic rays with individual element resolution, CALET is also carrying out direct measurements of the spectra and relative abundances of light and heavy cosmic nuclei $[3,4]$, from proton to iron, in the energy interval from $\sim 50 \mathrm{GeV}$ (for the lighter nuclei, $10 \mathrm{GeV} / n$ for the heavier) to several hundred $\mathrm{TeV}$. The abundances of trans-iron elements up to $\mathrm{Z} \sim 40$ are studied with a dedicated program of long term observations [5].

\section{THE CALET INSTRUMENT}

CALET is based on a thick calorimetric instrument (30 radiation lengths), designed to achieve electromagnetic shower containment and a large proton rejection capability $\left(>10^{5}\right)$. It is longitudinally segmented into a fine grained imaging calorimeter (IMC) followed by a total absorption calorimeter (TASC). The TASC is a $27 \mathrm{X}_{0}$ thick homogeneous calorimeter with 12 alternate $\mathrm{X}-\mathrm{Y}$ layers of lead-tungstate (PWO) logs. The IMC is a sampling calorimeter segmented longitudinally into 16 layers of scintillating fibers (with $1 \mathrm{~mm}^{2}$ square cross-section), readout individually, and interspaced with thin tungsten absorbers. Alternate planes of fibers are arranged along orthogonal directions. It can image the early shower profile in the first $3 \mathrm{X}_{0}$ and reconstruct the incident direction of cosmic rays with good angular resolution ( $0.1^{\circ}$ for electrons and better than $0.5^{\circ}$ for hadrons) [1]. The overall thickness of CALET at normal incidence is $\sim 1.3$ proton interaction lengths $\left(\lambda_{I}\right)$. The charge identification of individual nuclear species is performed by a two-layered hodoscope of plastic scintillators (CHD), positioned at the top of the apparatus, providing a measurement of the charge $Z$ of the incident particle over a wide dynamic range $(Z=1$ to $\sim 40)$ with sufficient charge resolution to resolve individual elements [6] and complemented by a redundant charge determination via multiple $\mathrm{dE} / \mathrm{dx}$ measurements in the IMC. The overall $\mathrm{CHD}$ charge resolution (in $\mathrm{Z}$ units) increases linearly, as a function of the atomic number, from $<0.1$ for protons to $\sim 0.3$ for iron. For the IMC, although the photostatistics available for a single fiber is about one order of magnitude lower than in the case of a single CHD layer, the charge measurement - thanks to the multiple sampling - achieves an excellent performance as shown in Fig.2 of [7], where the IMC charge resolution is plotted as a function of the atomic number $\mathrm{Z}$. The interaction point (IP) is first 
reconstructed [8] and only the $\mathrm{dE} / \mathrm{dx}$ ionization clusters from the layers upstream the IP are used to infer a charge value from the truncated-mean of the valid samples. The geometrical factor of CALET is $\sim 0.1 \mathrm{~m}^{2} \mathrm{sr}$ and the total weight is $613 \mathrm{~kg}$. The instrument is described in more detail elsewhere [9], [10].

\section{FLIGHT OPERATIONS AND CALIBRATIONS}

The commissioning of CALET aboard the ISS was successfully completed at the beginning of October 2015. Since then the instrument has been taking science data continuously with no major interruptions [2]. The on-orbit operations are controlled via the JAXA Ground Support Equipment (JAXA-GSE) in Tsukuba by the Waseda CALET Operations Center (WCOC) at Waseda University, Tokyo. As of April 30, 2021 a total observation time of more than 2027 days was integrated with a live time fraction $\sim 85 \%$ of the total time, and $\sim 2.7$ billion events were collected above $1 \mathrm{GeV}$. An exposure of $\sim 178 \mathrm{~m}^{2}$ sr day was achieved with the high-energy (HE) trigger mode, designed to maximize the collection power for electrons above $10 \mathrm{GeV}$ and other high-energy shower events. Energy calibrations of each channel of CHD, IMC, and TASC are performed with penetrating proton and $\mathrm{He}$ particles selected in-flight by a dedicated trigger mode. Raw signals are corrected for light output non-uniformity, gain differences among the channels, position and temperature dependence, as well as temporal gain variations $[9,11]$. The four gain ranges of each TASC channel are calibrated with flight data and linked together to provide a seamless response spanning more than six orders of magnitude and allowing observations from one minimum ionizing particle to $\mathrm{PeV}$ showers.

\section{RESULTS}

\subsection{Precision Measurement of the Electron Spectrum}

The new results on the analysis of electrons (+ positrons) with CALET presented at this conference [12] (see also [13], and the interpretation paper [14]) are based on 1815 days of flight data collected with the high-energy shower trigger. The flux shown in Fig.1 corresponds to an increase by a factor of 2.3 of the available statistics with respect to the latest CALET all-electron spectrum publication [15] and covers an energy range from $11 \mathrm{GeV}$ to $4.8 \mathrm{TeV}$. The error bars along the horizontal and vertical axes are representative of the bin width and statistical errors, respectively. The gray band bounds the quadrature sum of statistical and systematic errors. CALET design is optimized for the observation of possible narrow structures in the all-electron spectrum. The 30 $X_{0}$-thick calorimeter allows for a full containment of electron showers even at the $\mathrm{TeV}$ scale, with an excellent energy resolution ( $<2 \%$ above $20 \mathrm{GeV}$ ), while proton showers of equivalent energy deposit undergo a larger energy leakage from the bottom layers of the TASC. This feature is exploited to separate electrons from protons, building upon the capability of the TASC and IMC to image the longitudinal and lateral profiles of electromagnetic and hadronic cascades. Two methods were applied to identify electrons and to study systematic uncertainties in the electron identification: a simple two-parameter cut and a multivariate analysis based on boosted decision trees (BDTs). In the final electron sample, the residual contamination of protons is $\sim 5 \%$ up to $1 \mathrm{TeV}$, and $10 \%-20 \%$ in the $1-4.8 \mathrm{TeV}$ region, while keeping a constant high efficiency of $80 \%$ for electrons.

Systematic uncertainties include the absolute normalization error as well as energy dependent 


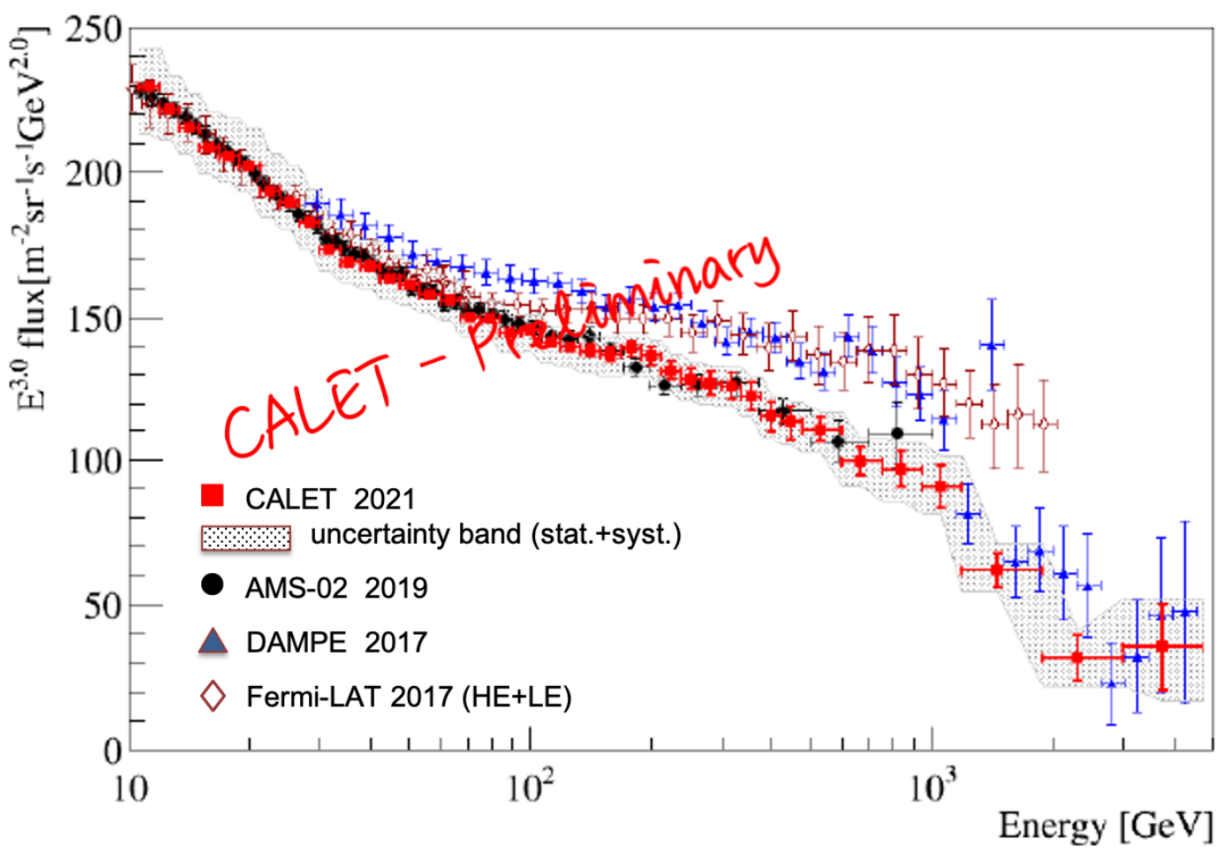

Figure 1: Cosmic-ray all-electron spectrum measured by CALET from $11 \mathrm{GeV}$ to $4.8 \mathrm{TeV}$ using the same energy binning as in our previous publication [15], where the gray band indicates the quadrature sum of statistical and systematic errors (not including the uncertainty on the energy scale). Also plotted are direct measurements in space [16-19] for comparison.

errors stemming from BDT stability, trigger efficiency in the low-energy region, tracking performance, dependence on charge and electron identification methods, and MC model dependence. Conservatively, all of them are included in the total error estimate of Fig.1. The absolute energy scale was calibrated and shifted by $+3.5 \%$ as a result of studies of the geomagnetic cutoff energy. The systematic uncertainties are described in more detail in $[10,15]$ and Supplemental Material therein.

Taking the currently available experimental data at face-value, we notice that the all-electron spectral data fork into two groups of measurements: AMS-02 + CALET and Fermi/LAT + DAMPE, with good consistency within each group, but with only marginal overlap between the two, possibly indicating the presence of unknown systematic errors. CALET spectrum is consistent with AMS-02 below $\sim 1 \mathrm{TeV}$ where both experiments have a good electron identification capability, albeit using different detection techniques. CALET observation of a flux suppression above $\sim 1 \mathrm{TeV}$ has now reached a significance $>6.5 \sigma$ and, in this region, it is found to be consistent with DAMPE within errors.

\subsection{Preliminary proton spectrum}

A preliminary measurement of the proton spectrum with CALET was presented at this conference [20], based on a restricted dataset collected within a fiducial geometrical acceptance (510 $\mathrm{cm}^{2} \mathrm{sr}$ ), during 1539 days of operation with the High Energy (HE) trigger and 27.9 days with the Low Energy trigger (LE), respectively. It is an update of the published CALET proton spectrum [21] based on a smaller statistical sample (1054 days) and limited to $10 \mathrm{TeV}$. The energy range was 
extended as shown in Fig. 2 where the preliminary proton spectrum spans the energy region from $30 \mathrm{GeV}$ to $60 \mathrm{TeV}$.

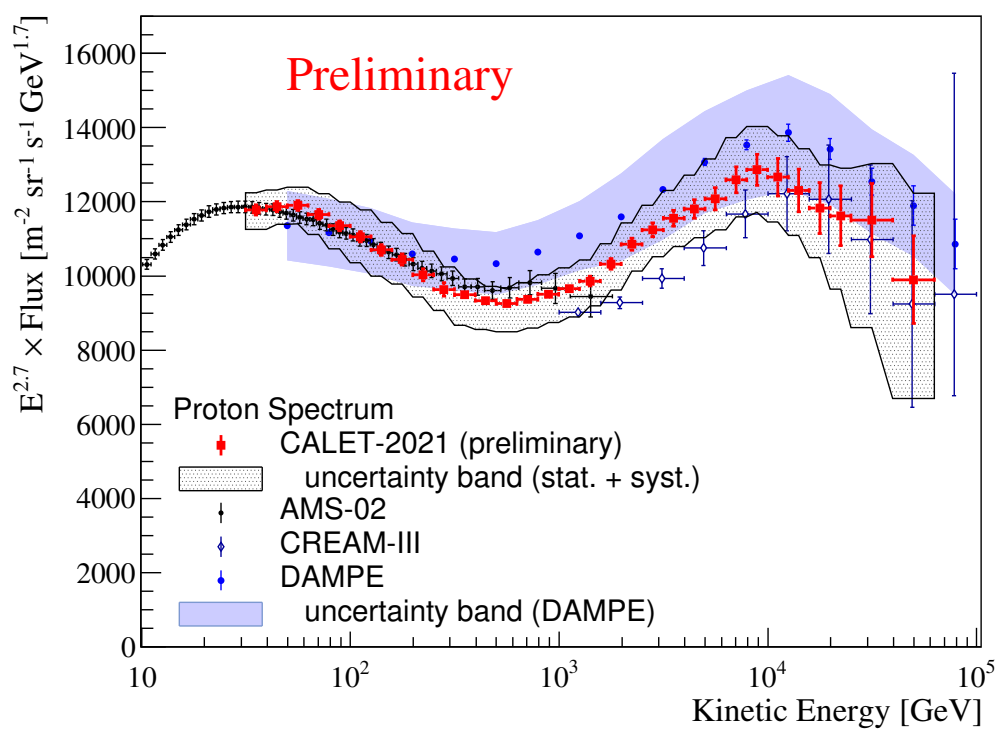

Figure 2: Preliminary proton spectrum measured by CALET (red circles) compared with the experimental results of AMS02 [22], CREAM-III [23], and DAMPE [24]. The hatched band shows the total uncertainty for CALET. The dark blue colored band shows the total uncertainty for DAMPE.

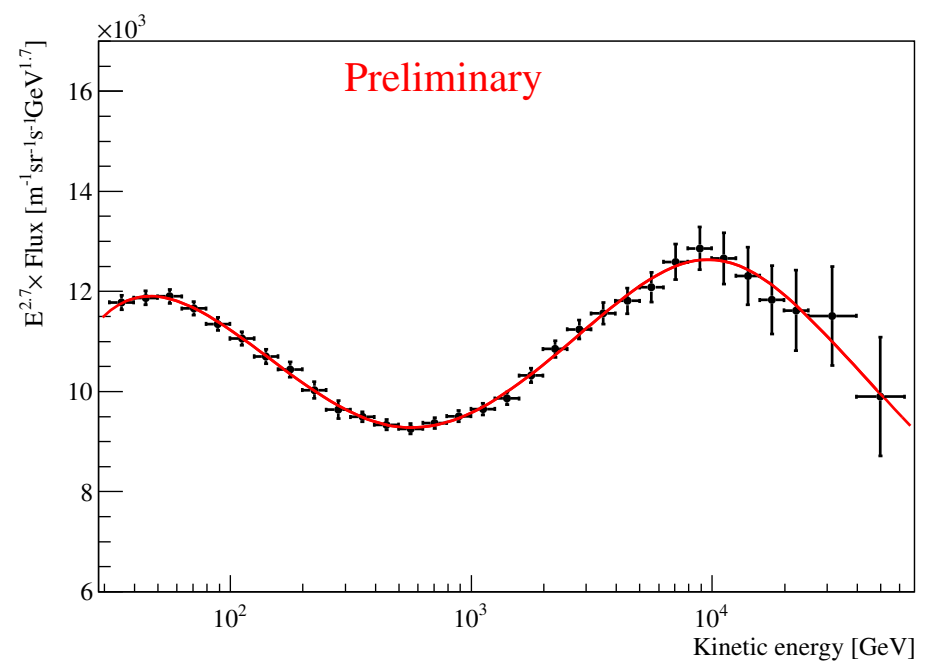

Figure 3: A fit of the CALET proton spectrum (solid red line) with a Double Broken Power Law (eq.1). The horizontal error bars are representative of the bin width.

In the same figure the CALET flux is compared with AMS-02, CREAM-III, and DAMPE. In the low energy region with $E<200 \mathrm{GeV}$, the result is fully consistent. In the higher energy region, a systematic difference is observed, but the difference is within the errors. We confirm the presence of a spectral hardening around $500 \mathrm{GeV}$ as reported in [21]. We also observe a spectral softening 
around $10 \mathrm{TeV}$. We cross-checked two independent CALET analyses with different efficiencies and the two results were found to be consistent. In order to quantify the spectral hardening and softening, we fit the proton spectrum using a Double Broken Power Law (DBPL) function defined as follows:

$$
\Phi^{\prime}=E^{2.7} \times C \times\left(1-\frac{p_{0}}{E}-\frac{p_{1}}{E^{2}}\right) \times\left(\frac{E}{45}\right)^{\gamma} \times\left[1+\left(\frac{E}{E_{0}}\right)^{s}\right]^{\frac{\Delta \gamma}{s}} \times\left[1+\left(\frac{E}{E_{1}}\right)^{s}\right]^{\frac{\Delta \gamma_{1}}{s}}
$$

where $\Phi^{\prime}$ is the proton flux $\times E^{2.7}, C$ is a normalization factor, $p_{0}$ and $p_{1}$ are the fitting parameters for the low energy region, $s$ is the smoothness parameter, $\gamma$ the spectral index, $E_{0}$ the break energy at the onset of spectral hardening, $\Delta \gamma$ the spectral variation due to the spectral hardening, $E_{1}$ the softening break energy, $\Delta \gamma_{1}$ is spectral index variation observed above $E_{1}$.

In Fig.3, the black filled circles show the data with statistical errors and the red line shows the best fitted function. The $\chi^{2}$ is 2.9 with 22 degrees of freedom. The best fitted parameters are: $p_{0}=9.1 \pm 26, p_{1}=-6.6 \pm 470, \gamma=-2.9 \pm 0.3, s=2.1 \pm 2.0, \Delta \gamma=(4.4 \pm 3.8) \times 10^{-1}$, $E_{0}=(5.5 \pm 1.3) \times 10^{2} \mathrm{GeV}, \Delta \gamma_{1}=(-4.4 \pm 3.0) \times 10^{-1}$, and $E_{1}=(1.1 \pm 0.4) \times 10^{4} \mathrm{GeV}$. The spectral hardening starts at $550 \pm 130 \mathrm{GeV}$ and the softening starts at $11 \pm 4 \mathrm{TeV}$.

\subsection{Preliminary helium spectrum}

A preliminary spectrum of CR helium was presented at this conference [25] as shown in figure 4 , where it is compared with previous observations from space-based [26, 27] and balloon-borne [28] experiments. This result was obtained with a dataset of limited statistics (1815 days) collected

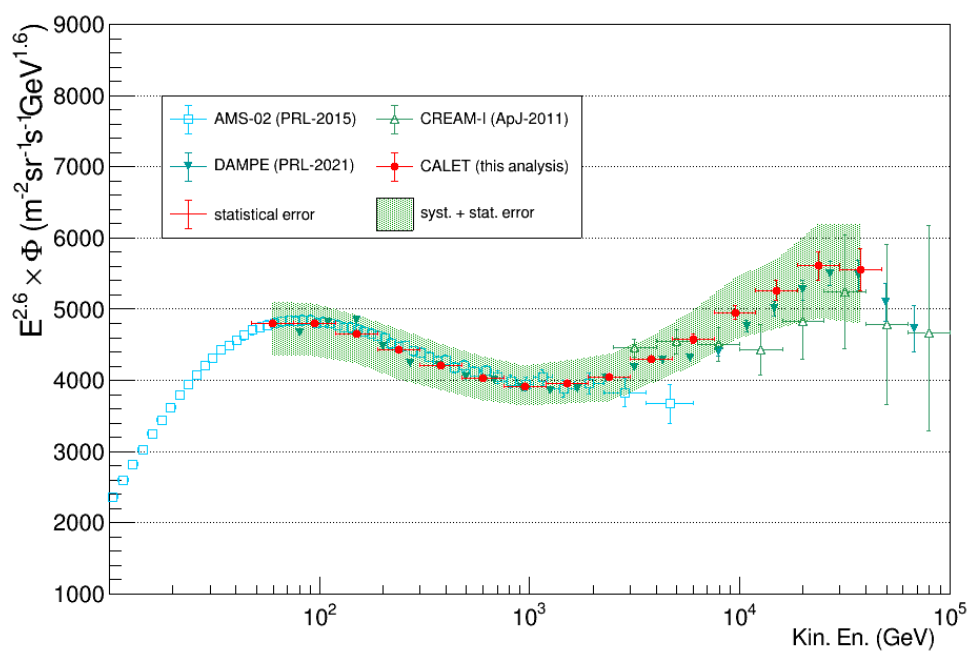

Figure 4: Preliminary helium flux measurement with CALET (red markers), compared with previous direct observations [26-28]. The error bars are the statistical errors, the green band is representative of the combined statistical and systematic uncertainties.

within a restricted "fiducial" geometrical acceptance $\left(\sim 510 \mathrm{~cm}^{2} \mathrm{sr}\right)$ which amounts to about one half of the total acceptance.

The energy sub-ranges $[80,600] \mathrm{GeV}$ and $[2,20] \mathrm{TeV}$ can be fitted individually with a single power law function, but not the whole interval. Instead, a progressive hardening is observed up to the 

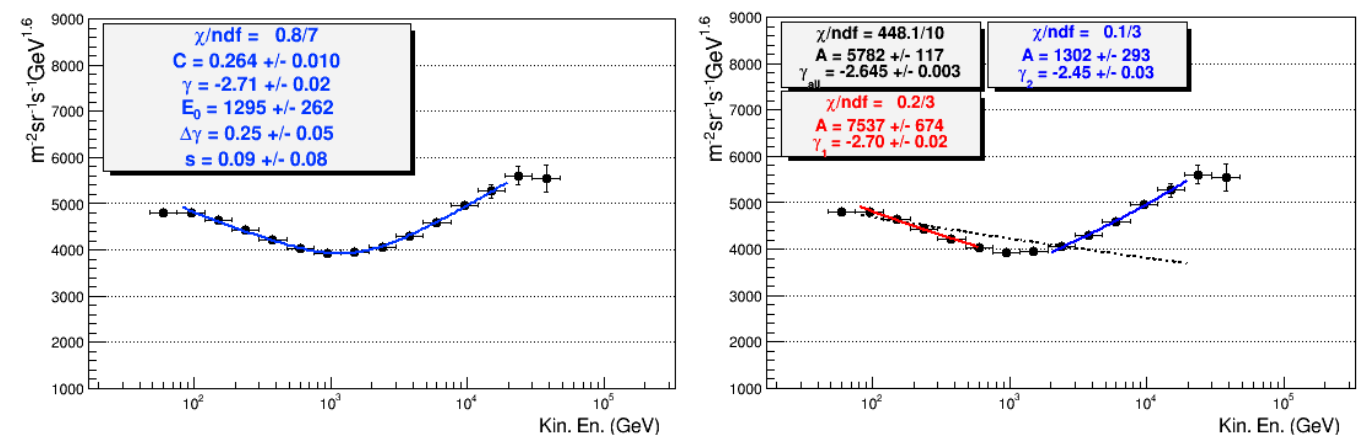

Figure 5: Left panel: a "Smoothly Broken Power-Law" [26] function is used for the fit and the result is consistent with DAMPE [27] within the errors. Right panel: fit to a single power low function in the two sub-ranges $[80,600] \mathrm{GeV},[2,20] \mathrm{TeV}$ and in the full range. The sub-ranges are individually compatible with a single power law function, the whole range is not.

multi-TeV region and fitted to a "Smoothly Broken Power-Law" (SBPL) function [26]. The fit (left panel of figure 5) gives a power law index $\gamma=-2.71 \pm 0.02, \Delta \gamma=0.25 \pm 0.05$ and break energy $\left(E_{0}\right)$ $=1295 \pm 262 \mathrm{GeV}$ consistent, within the errors, with the most recent results of DAMPE [27]. The detailed study of systematic uncertainties can be found in [25]. The preliminary results from two independent helium analyses, carried out by separate groups inside the CALET collaboration and using different event selections and background rejection procedures, were found to be consistent within the errors.

\subsection{Carbon and oxygen fluxes}

The spectra of cosmic-ray carbon and oxygen and their ratio were published by CALET in [29] and presented at this conference [30]. They are shown in Fig. 6 in an energy range from $10 \mathrm{GeV} / n$ to $2.2 \mathrm{TeV} / n$ where uncertainties, including statistical and systematic errors, are represented by a gray band. CALET carbon spectrum is consistent with PAMELA [36] and with most previous experiments $[31-34,37]$. No oxygen spectrum was published by PAMELA. AMS-02 carbon and oxygen spectra differ in the absolute normalization, which is lower for CALET by about $27 \%$ for both $\mathrm{C}$ and $\mathrm{O}$. However they have very similar shapes as confirmed by the good consistency of the respective measurements of the $\mathrm{C} / \mathrm{O}$ flux ratio.

Fig.7 shows the fits to CALET carbon and oxygen data (blue solid line) with a Double Power-Law (DPL) function:

$$
\Phi(E)= \begin{cases}C\left(\frac{E}{\mathrm{GeV}}\right)^{\gamma} & E \leq E_{0} \\ C\left(\frac{E}{\mathrm{GeV}}\right)^{\gamma}\left(\frac{E}{E_{0}}\right)^{\Delta \gamma} & E>E_{0}\end{cases}
$$

where $C$ is a normalization factor, $\gamma$ the spectral index, and $\Delta \gamma$ the spectral index change above the transition energy $E_{0}$. A single power-law (SPL) function (Eq.2 with $\Delta \gamma=0$ ), fitted to the data in the energy range $[25,200] \mathrm{GeV} / n$ and extrapolated above $200 \mathrm{GeV} / n$, is also shown for comparison (dashed line).

The effect of systematic uncertainties in the measurement of the energy spectrum is modeled in the $\chi^{2}$ minimization function with a set of 6 nuisance parameters [29]. The DPL fit to the $\mathrm{C}$ spectrum yields a spectral index $\gamma=-2.663 \pm 0.014$ at energies below the transition region 


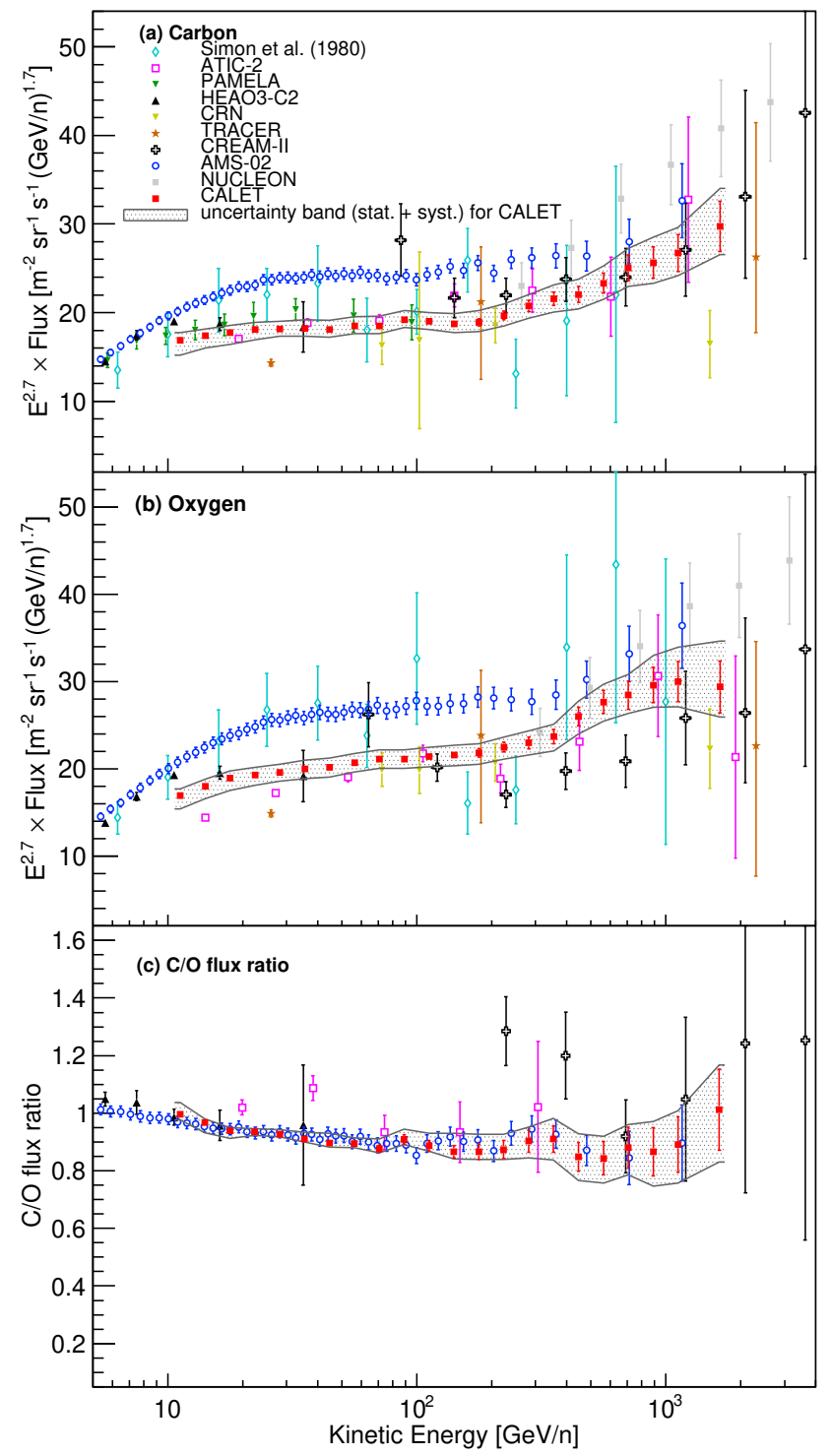

Figure 6: CALET (a) carbon and (b) oxygen flux (multiplied by $E^{2.7}$ ) and (c) ratio of carbon to oxygen fluxes, as a function of kinetic energy $E$. Error bars of CALET data (red filled circles) represent the statistical uncertainty only, while the gray band indicates the quadratic sum of statistical and systematic errors. Also plotted are other direct measurements [31-39].

$E_{0}=(215 \pm 54) \mathrm{GeV} / n$ and a spectral index increase $\Delta \gamma=0.166 \pm 0.042$ above, with $\chi^{2} /$ d.o.f. $=$ $9.0 / 8$. For oxygen, the fit yields $\gamma=-2.637 \pm 0.009, E_{0}=(264 \pm 53) \mathrm{GeV} / n, \Delta \gamma=0.158 \pm 0.053$, with $\chi^{2} /$ d.o.f. $=3.0 / 8$. The SPL fits gives $\gamma=-2.626 \pm 0.010$ with $\chi^{2} /$ d.o.f. $=27.5 / 10$ for $\mathrm{C}$, and $\gamma=-2.622 \pm 0.008$ with $\chi^{2} /$ d.o.f. $=15.9 / 10$ for $\mathrm{O}$, respectively. A frequentist test statistic $\Delta \chi^{2}$ is computed from the difference in $\chi^{2}$ between the fits with SPL and DPL functions. For carbon (oxygen), $\Delta \chi^{2}=18.5$ (12.9) with 2 d.o.f. (i.e. the number of additional free parameters in DPL fit with respect to SPL fit) implies that the significance of the hardening of the $\mathrm{C}(\mathrm{O})$ spectrum exceeds the $3 \sigma$ level.

In order to study the energy dependence of the spectral index in a model independent way, the 

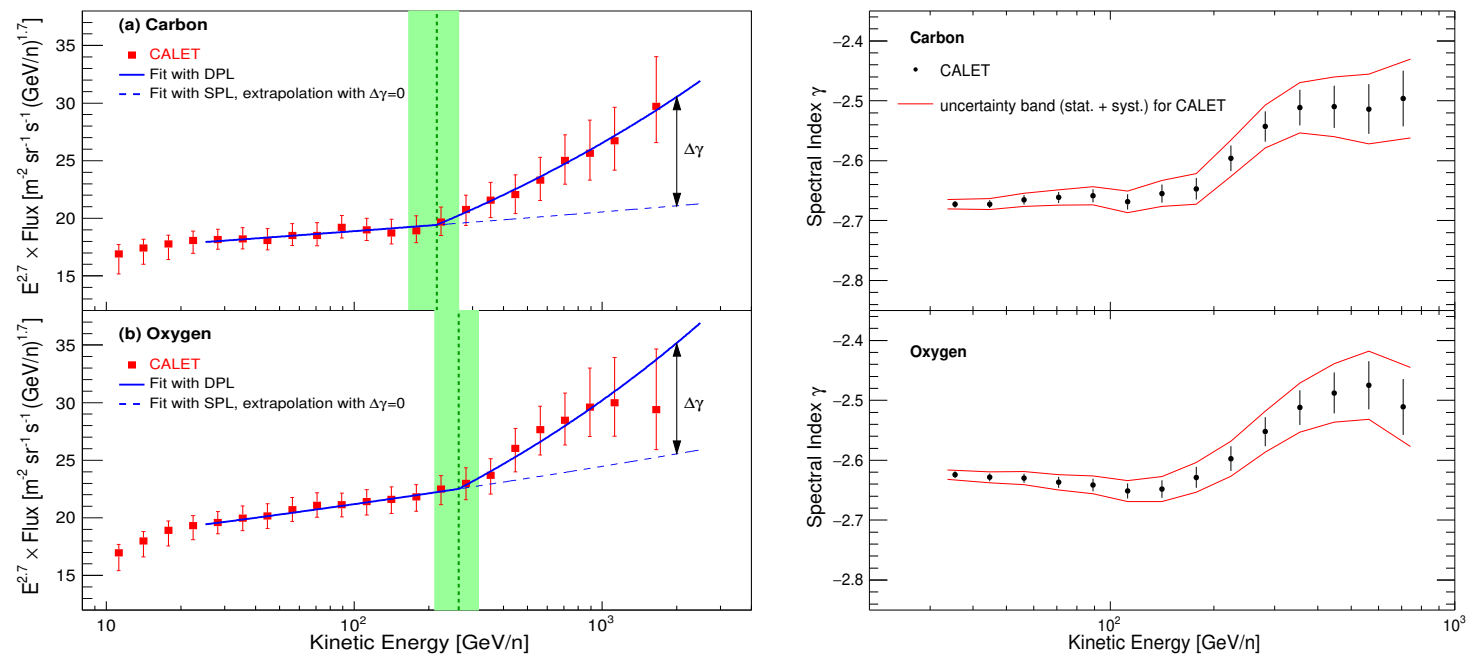

Figure 7: Left panel: Fit of the CALET (a) $\mathrm{C}$ and (b) O energy spectra with a DPL function (Eq.2, blue line) in the energy range [25, $2000] \mathrm{GeV} / n$. The flux is multiplied by $E^{2.7}$ where $E$ is the kinetic energy per nucleon. Error bars of CALET data points represent the sum in quadrature of statistical and systematic uncertainties. The dashed blue lines represent the extrapolation of a SPL function fitted to data in the energy range $[25,200] \mathrm{GeV} / n . \Delta y$ is the change of the spectral index above the transition energy $E_{0}$, represented by the vertical green dashed line. The green band is representative of the uncertainty on $E_{0}$ from the DPL fit.

Right panel: Energy dependence of the spectral index calculated within a sliding energy window for CALET (a) C and (b) O data. The spectral index is determined for each bin by fitting the data using \pm 3 bins. Red curves indicate the uncertainty range including systematic errors.

spectral index $\gamma$ is calculated via a fit of $d[\log (\Phi)] / d[\log (E)]$ in energy windows centered in each bin and including the neighbor \pm 3 bins. The results in Fig. 7 show that carbon and oxygen fluxes harden in a similar way above a few hundred $\mathrm{GeV} / n$. The carbon to oxygen flux ratio is well fitted to a constant value of $0.911 \pm 0.006$ above $25 \mathrm{GeV} / n$, confirming that the two fluxes have the same energy dependence [29].

\subsection{Preliminary boron flux and $\mathrm{B} / \mathrm{C}$ ratio}

Preliminary CALET measurements of cosmic-ray boron flux and boron to carbon ratio were presented at this conference [40].

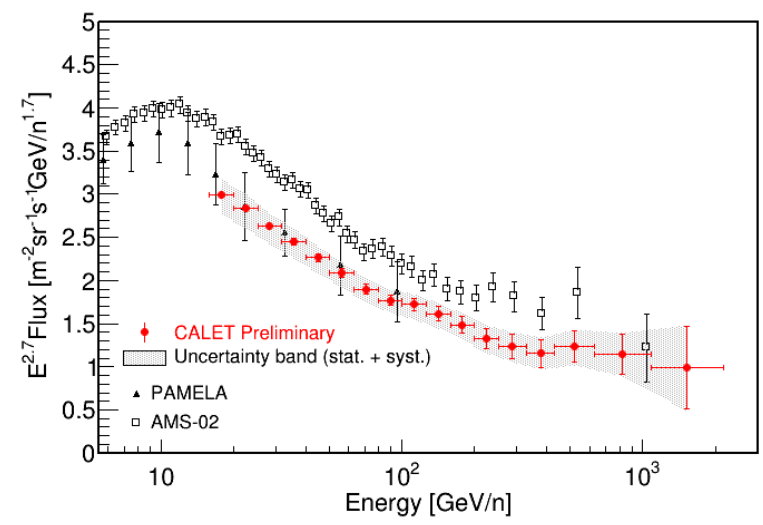

Figure 8: Preliminary energy spectrum of boron as a function of kinetic energy per nucleon with CALET compared with previous observations [41, 42]. 
The boron spectrum is shown in Fig. 8 as a function of kinetic energy per nucleon from $16 \mathrm{GeV} / n$ to $2.2 \mathrm{TeV} / n$ and compared with PAMELA [41] and AMS-02 [42]. The isotope composition ${ }^{11} \mathrm{~B} /\left({ }^{10} \mathrm{~B}+{ }^{11} \mathrm{~B}\right)$ is assumed to be 0.7 . Different values of the isotope ratio $(0.6$ and 0.8$)$ make only a $2 \%$ difference in the boron spectrum. CALET result is well consistent with PAMELA, but lower than AMS-02 as in the cases of carbon, oxygen and iron spectra [29, 43]. Fig. 9 shows a preliminary measurement of the $\mathrm{B} / \mathrm{C}$ ratio as a function of kinetic energy per nucleon from $16 \mathrm{GeV} / n$ to $2.2 \mathrm{TeV} / n$ compared with the previous observations [41, 42, 44-48]. CALET result is well consistent with previous measurements such as CREAM-I, PAMELA and AMS-02. Our preliminary $\mathrm{B} / \mathrm{C}$ ratio can be fit with a single power law $(\mathrm{B} / \mathrm{C})=A E^{-\delta}$, where $\mathrm{A}$ is a normalization constant. In the energy interval $25 \mathrm{GeV} / n-100 \mathrm{GeV} / n, \delta=0.406 \pm 0.039$ with $\chi^{2} / n d f=0.30 / 3$, while in the interval $100 \mathrm{GeV} / n-2.2 \mathrm{TeV} / n$ we get $\delta=0.366 \pm 0.064$ with $\chi^{2} / n d f=1.2 / 7$. The $\mathrm{B} / \mathrm{C}$ ratio is consistent with AMS-02 and other experiments, and the $\delta$ coefficient values below and above $100 \mathrm{GeV} / n$ are in agreement with AMS-02 within the errors[42].

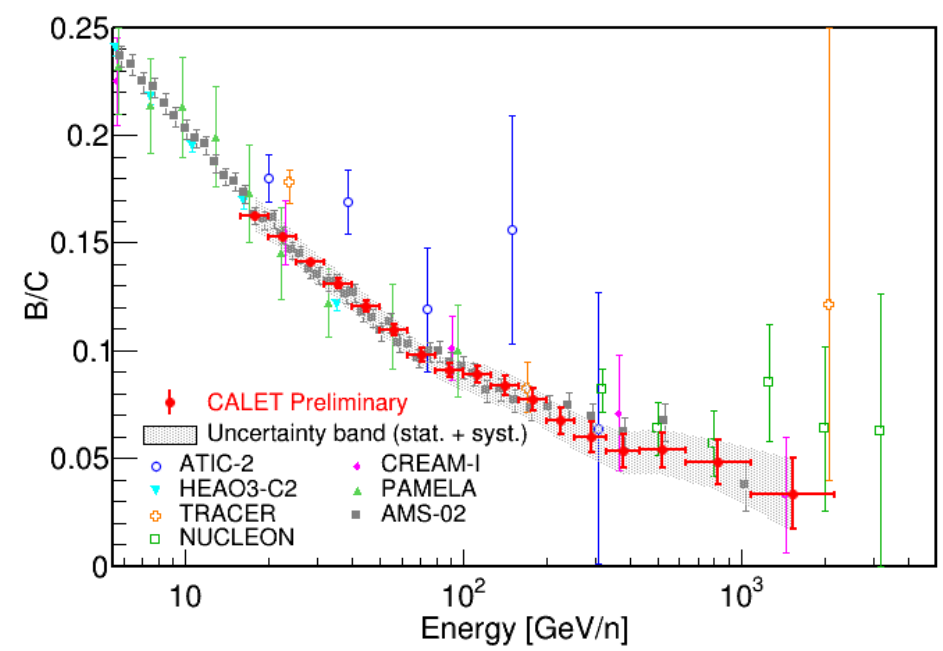

Figure 9: Preliminary result of the boron-to-carbon ratio as a function of kinetic energy with CALET (red filled points) compared with previous observations [41, 42, 44-48].

\subsection{Iron flux}

The spectrum of cosmic-ray iron was recently published by CALET [43] and presented at this conference [49]. The iron flux (multiplied by $E^{2.6}$ ) is shown in Fig.10 (red filled circles) in the interval $10 \mathrm{GeV} / n$ to $2.0 \mathrm{TeV} / n$. In comparison with other recent measurements, the CALET iron spectrum is consistent with ATIC02 [50] and TRACER [51] at low energy, and with CRN [52] and HESS [53] at high energy, while the absolute normalization is higher than NUCLEON [54] and lower than Sanriku [55]. AMS-02 [56] iron spectrum has a 20\% higher absolute normalization than CALET flux. However, when the latter is multiplied by $E^{2.7}$ (as in [56]) and normalized to the AMS-02 flux, the two spectra show a very similar shape and comparable errors (more details in the Supplemental Material of [43]).

Fig. 11 shows a fit to a single power law (SPL) function from $50 \mathrm{GeV} / n$ to $2.0 \mathrm{TeV} / n$. The fit gives a spectral index $\gamma=-2.60 \pm 0.02$ (stat) \pm 0.02 (sys) with $\chi^{2} / D O F=4.2 / 14$. The result is stable when the binning is changed from 10 to 4 bins/decade $(\gamma=-2.59 \pm 0.02($ stat $) \pm 0.04($ sys $))$. 


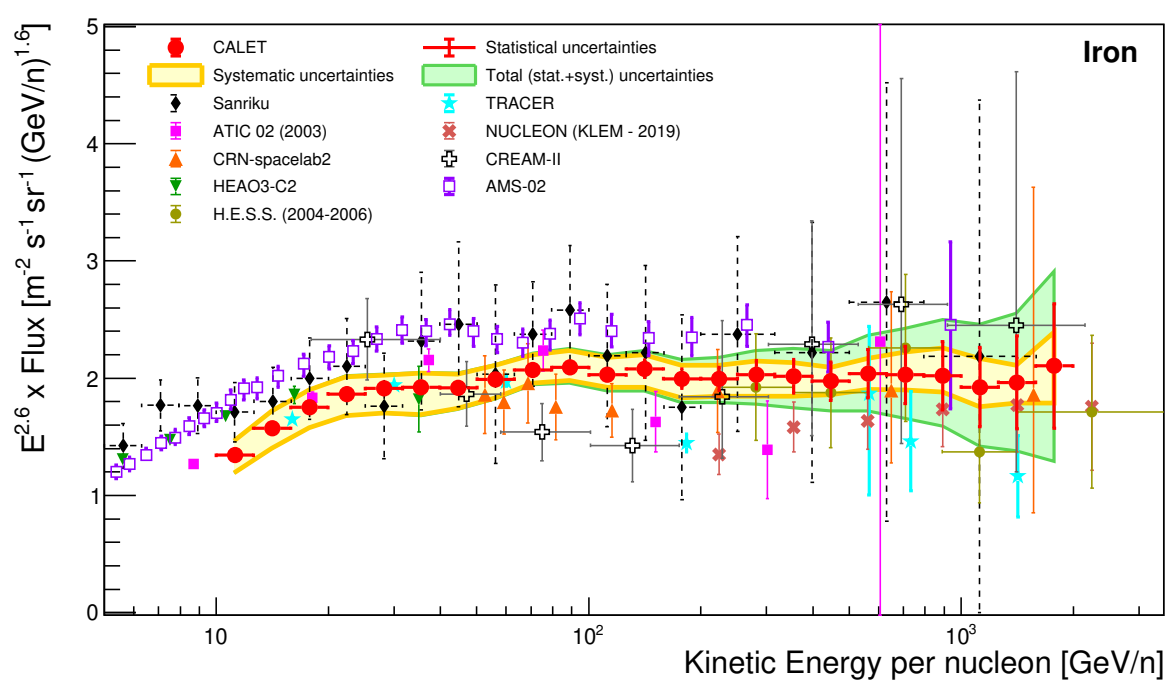

Figure 10: CALET iron flux (multiplied by $E^{2.6}$ ) as a function of kinetic energy per nucleon (red filled circles). The error bars represent the statistical uncertainty only, the yellow band indicates the quadrature sum of systematic errors, while the green band bounds the quadrature sum of statistical and systematic errors. Also plotted are the data points from other direct measurements [50-58]. The horizontal error bars are representative of the bin width.

The energy dependence of the spectral index $\gamma$ is also calculated by a fit of $d[\log (\Phi)] / d[\log (E)]$ inside a sliding window centered in each energy bin and including the neighboring \pm 3 bins. The results in Fig.11 show that the iron flux is compatible, within the errors, with a single power law in the fit region between $50 \mathrm{GeV} / n$ and $2 \mathrm{TeV} / n$.

The systematic error related to charge identification was studied by varying the semiaxes of the elliptical selection resulting in a flux variation lower than a few percent below $600 \mathrm{GeV} / n$. Using FLUKA (instead of EPICS [59]) as simulation code, the resulting fluxes show a maximum discrepancy around $10 \%$ below $40 \mathrm{GeV}$.
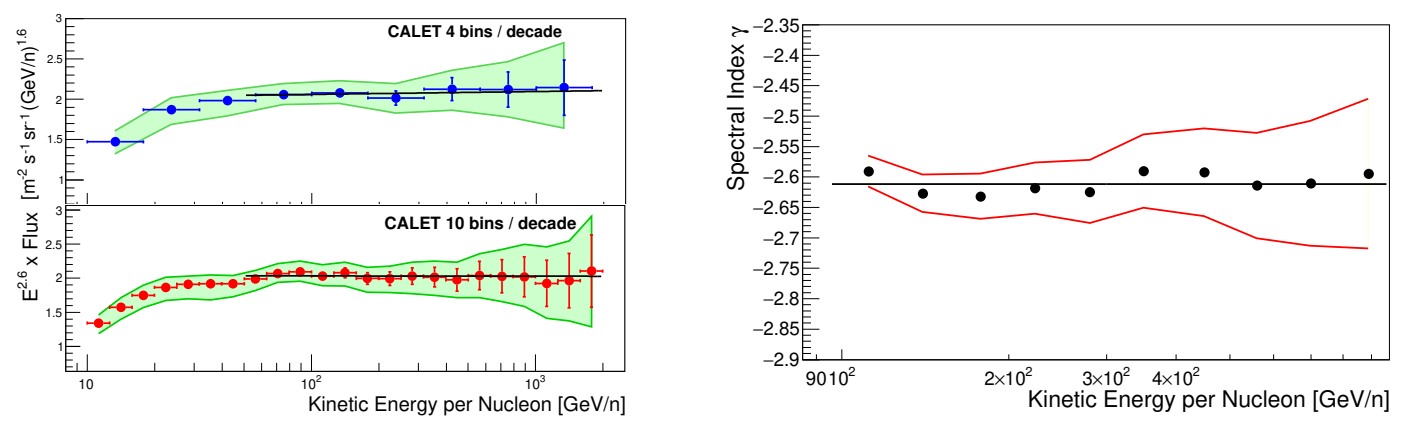

Figure 11: (left): Fit of the CALET iron energy spectrum to a SPL function (black lines) in the energy range [50, 2000] $\mathrm{GeV} / n$ with 4 bins/decade (top) and 10 bins/decade (bottom).The green band indicates the quadrature sum of statistical and systematic errors; (right): Energy dependence of the spectral index calculated within a sliding energy window. The fit with a constant function gives a mean spectral index value $\langle\gamma\rangle=-2.61 \pm 0.01$.

The uncertainty on the energy scale correction is $\pm 2 \%$ and causes a rigid shift of the measured energies, affecting the absolute flux normalization by ${ }_{-3.2 \%}^{+3.3 \%}$, but not the spectral shape. The uncertainties due to the unfolding procedure were evaluated with different response matrices computed by varying the spectral index (between -2.9 and -2.2) of the MC generation spectrum, or by using the Singular Value Deconvolution method, instead of the Bayesian approach. The contributions 
due to the beam test model (not identical to the instrument now in orbit) and to the shower event cut were evaluated and included in the systematic uncertainties [43]. The systematic errors due to off-acceptance events, tracking efficiency, background contamination and HE trigger efficiency are negligible. Energy-independent systematic uncertainties affecting the flux normalization include live time $(3.4 \%)$, long-term stability $(<2 \%)$, and geometrical factor $(\sim 1.6 \%)$.

\subsection{Preliminary results on Ultra Heavy Cosmic Rays}

Ultra Heavy Cosmic Rays (UHCR) with charges $30 \leq \mathrm{Z} \leq 40$ are $\sim 10^{5}$ less abundant than iron. In order to collect these rare events, CALET implements a special ultra-heavy trigger which provides an increased acceptance by requiring event trajectories to traverse the CHD and the top half of the IMC. This corresponds to an acceptance angle of $\pm 75^{\circ}$, which gives an enhanced geometry factor of $\sim 4400 \mathrm{~cm}^{2} \mathrm{sr}$ (almost $\times 4$ the total acceptance with the HE trigger).
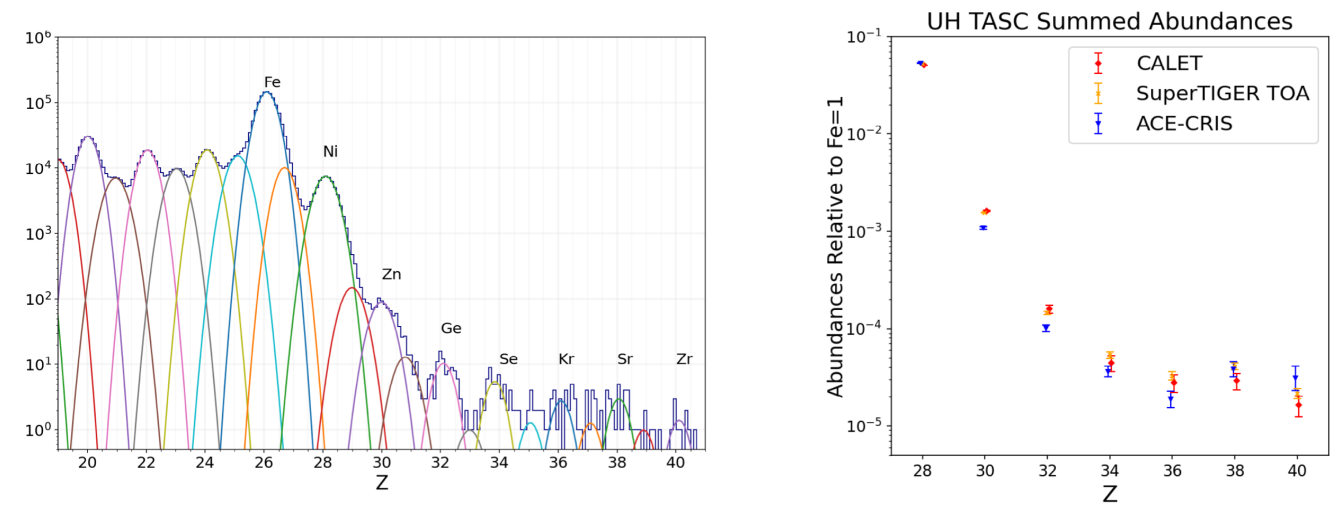

Figure 12: (left): UHCR TASC analysis histogram with multiple gaussian fitting, bin size in 0.1 units of charge; (right): Comparison of the relative abundances of the summed odd-even pairs with ACE-CRIS[63] and SuperTIGER [66] for Z between $27 \leq \mathrm{Z} \leq 40$. Errors bars are statistical only.

To date, single-element resolution measurements of UHCR have only been made by TIGER [60] (up to ${ }_{40} \mathrm{Zr}$ ) and by SuperTIGER [61, 62] (up to ${ }_{56} \mathrm{Ba}$ ) balloon-borne instruments at $\mathrm{GeV} / n$ energies, as well by the ACE-CRIS [63] space based instrument (up to ${ }_{40} \mathrm{Zr}$ ) at lower energies (hundreds of $\mathrm{MeV} / \mathrm{n}$ ). The balloon based observations must be corrected for energy losses and nuclear interactions in the atmosphere, while ISS based measurements are subject to screening by the Earth's geomagnetic field. Comparatively, UHCR observations made by ACE-CRIS (sitting near the L1 libration point) occur outside the geomagnetic field and cover a complementary lower energy range. While ACE-CRIS and SuperTIGER instruments only measure charges down to $\mathrm{B}$ and $\mathrm{Ne}$, respectively, CALET measures cosmic-ray abundances in the $1 \leq \mathrm{Z} \leq 40$ charge range providing complementary measurements and a check of the cross calibrations of other instruments. Improved analyses of CALET UHCR, based on 5 years of data, were presented at this conference $[64,65]$. The first one analyzes the smaller dataset of events that pass through the TASC, thus providing energy information and a better charge assignment, albeit with lower statistics. The second one implements a new method [65] based on trajectory tracing to compute effective cutoff rigidities in place of the previously used vertical Störmer approximation. The results shown in Fig.12 are consistent with previous CALET findings [67] based on a smaller statistics, and are in 
good agreement with SuperTIGER. The lower energy interval covered by ACE-CRIS has to be taken into account when comparing with CALET data. A preliminary comparison shows an approximate agreement.

\subsection{Further CALET Observations}

Solar Modulation CALET observations of low-energy CRs have been successfully performed with a Low-Energy Electron (LEE) shower trigger mode activated only at high geomagnetic latitudes. An analysis of low-energy electrons and protons to investigate the solar modulation during the descending phase of the solar cycle 24 was presented at this conference [68]. CALET data confirm that, during the solar minimum, the flux of the low-energy electrons has reached its maximum, which is comparable to or exceeding the maximum flux observed with PAMELA in the previous solar minimum period.

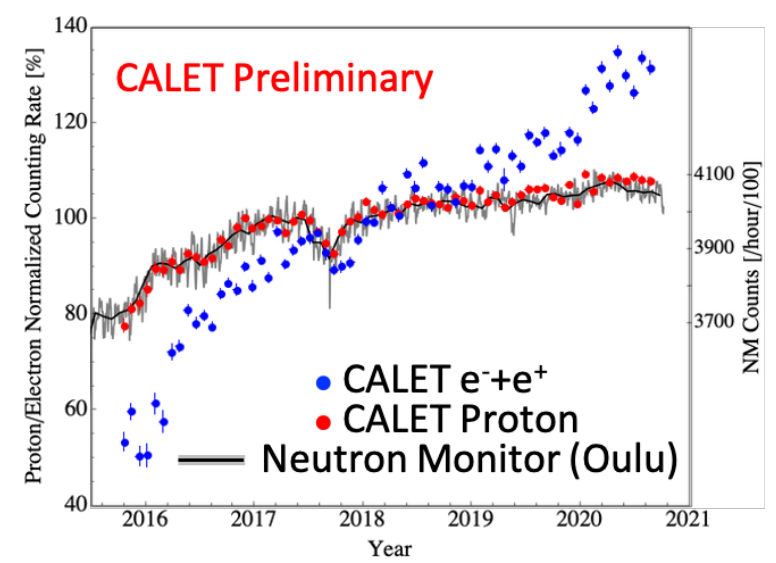

Figure 13: Normalized counting rates for proton (red filled circles) and electron+positron (blue filled circles) as a function of time. The NM counting rate at Oulu station (black points and vertical scale on the right end side of the figure) shows a good correlation with the $\mathrm{CR}^{-}+\mathrm{e}^{+}$flux increase in the $1-10 \mathrm{GeV}$ energy interval until about half a year after the beginning the new solar cycle 25. The flux has now started decreasing.

The count rates of electrons and protons (Fig.13), at the same average rigidity, show a clear charge sign dependence as expected from the drift model of the solar modulation. More details can be found in [68].

Gamma-rays observations CALET is sensitive to gamma rays from $1 \mathrm{GeV}$ up to $10 \mathrm{TeV}$, limited by statistics. Access to energies below $10 \mathrm{GeV}$ is enabled by a dedicated low-energy gamma (LE- $\gamma$ ) trigger which is active only at low geomagnetic latitudes. The analysis of gamma-ray events collected with this trigger were presented at this conference [69] including the mitigation of a secondary photon background from cosmic-ray interactions with ISS structures in the CALET field-of-view, the observation of persistent galactic and extragalactic sources, and the detection of emission from the quiescent Sun.

Gamma-ray Transients, GW follow-up The CALET gamma-ray burst monitor (CGBM), designed to observe prompt emissions of gamma-ray bursts (GRBs) in the hard X-ray and soft gamma-ray band, has been providing all-sky monitoring, with $\sim 60 \%$ duty cycle and without any problems, since October 2015. As of the end of May 2021, the CGBM has detected 254 GRBs, including 31 short GRBs. CALET has actively participated in the follow-up campaign for the 
search of electromagnetic counterparts of the gravitational wave events observed by LIGO/Virgo. Although no candidates have been found, upper limits on the high-energy gamma-ray flux were derived for 26 events from the LIGO/Virgo third observation run. The above observations were presented at this conference [70].

Dark Matter limits Gamma-ray events above $10 \mathrm{GeV}$ have been analysed to search for possible line signals in the energy spectra, assuming various regions-of-interest on the sky based on specific assumptions derived from Galactic halo density profile models. In the analysis presented at this conference [71] no hint of line signals were reported. Upper limits on parameters of dark matter annihilation and decay models were evaluated although these limits, which take into account only statistical errors, are still preliminary.

Space Weather CALET provides a continuous monitoring of space-weather phenomena affecting the near-Earth environment, including solar energetic particle and relativistic electron precipitation (REP) events. In the work presented at this conference [72], preliminary results of the REP observations in a period of $\sim 4.5$ years, were shown together with the investigation on their correlations with the interplanetary and geomagnetic conditions.

\section{Summary}

CALET instrument performance on the ISS has been very stable during the whole scientific observation period from Oct. 13, 2015. At this conference, CALET presented new data based on about 2000 days of observation and $\sim 2.7$ billion triggers (for energies $>1 \mathrm{GeV}$ ). They include an updated measurement of the electron and positron spectrum up to $4.8 \mathrm{TeV}$ [12], [13], [14], a preliminary update of the proton spectrum [20] to $\sim 60 \mathrm{TeV}$, and a preliminary analysis of the helium spectrum to $\sim 50 \mathrm{TeV}$ [25]. Cosmic-ray carbon and oxygen and their flux ratio were published by CALET in [29] and presented at this conference [30] together with preliminary measurements of the boron flux and boron to carbon ratio [40] up to $2.2 \mathrm{TeV} / n$. The spectrum of cosmic-ray iron was recently published by CALET up to $2.0 \mathrm{TeV} / n$ [43] and presented at this conference [49]. Improved analyses of CALET UHCR were also presented [64, 65], as well as gamma-ray measurements [69], GRB observations and searches of GW events counterparts [70], DM searches[71], and Space Weather observations [72].

Extended CALET operations were approved by JAXA/NASA/ASI in March 2021 through the end of 2024 (at least). Improved statistics and refinement of the analysis with additional data collected during the livetime of the mission will allow to extend the measurements at higher energies and improve the spectral analysis, contributing to a better understanding of CR phenomena.

\section{Acknowledgments}

We gratefully acknowledge JAXA 's contributions to the development of CALET and to the operations aboard the JEM-EF on the International Space Station. We also wish to express our sincere gratitude to Agenzia Spaziale Italiana (ASI) and NASA for their support of the CALET project. This work was supported in part by JSPS Grant-in-Aid for Scientific Research (S) Number 26220708 and 19H05608, JSPS Grant-in-Aid for Scientific Research (B) Number 17H02901, JSPS Grant-in-Aid for Research Activity Start-up No.20K22352 and by the MEXT-Supported Program for the Strategic Research Foundation at Private Universities (20112015) (No.S1101021) at Waseda University. The CALET effort in Italy is supported by ASI under agreement 2013-018-R.0 and its amendments. The CALET effort in the United States is supported by NASA through Grants No. NNX16AB99G, No. NNX16AC02G, and No. NNH14ZDA001N-APRA-0075.

\section{References}

[1] S. Torii (CALET), in PoS (ICRC2019) 142 (2019).

[2] Y. Asaoka (CALET), in PoS (ICRC2019) 001 (2019).

[3] S. Torii and P. S. Marrocchesi (CALET), Adv. Space Res. 64, 2531 (2019) . 
[4] Y. Akaike (for the CALET Collab.), in PoS (ICRC2019) 034 (2019) .

[5] B. F. Rauch (for the CALET Collab.), in PoS (ICRC2019) 130 (2019).

$6]$ P. S. Marrocchesi, et al., Nucl. Instrum. Methods A 659, 477 (2011).

7 P. S. Marrocchesi (CALET), in PoS (ICRC2019) 103 (2019).

8] P. Brogi, et al., PoS (ICRC2015) 595 (2015)

$9]$ Y. Asaoka, et al. (CALET), Astropart. Phys. 91, 1 (2017)

10] O. Adriani et al. (CALET), Phys. Rev. Lett. 119, 181101 (2017).

11] Y. Asaoka, et al. (CALET), Astropart. Phys. 100, 29 (2018) .

12] S. Torii and Y. Akaike (CALET), in PoS (ICRC2021) 105 (2021).

$13]$ E. Berti (CALET), in PoS (ICRC2021) 065 (2021) .

14 H. Motz (CALET), in PoS (ICRC2021) 100 (2021).

15] O. Adriani et al. (CALET), Phys. Rev. Lett. 120, 261102 (2018) .

16] M. Aguilar et al. (AMS02), Phys. Rev. Lett. 122, 101101 (2019).

17 G. Ambrosi, et al. (DAMPE), Nature 552, 63 (2017)

18] O. Adriani et al. (PAMELA), Riv. Nuovo Cimento 40, 473 (2017) .

$19]$ S. Abdollahi et al. (Fermi-LAT), Phys. Rev. D 95, 082007 (2017).

20] K. Kobayashi and P.S. Marrocchesi (CALET), in PoS (ICRC2021) 098 (2021) .

21] O. Adriani et al. (CALET), Phys. Rev. Lett. 122, 181102 (2019).

$22]$ M. Aguilar et al. (AMS02), Phys. Rev. Lett. 114, 171103 (2015).

$23]$ Y. S. Yoon et al. (CREAM-III), Astrophys. J 839, 5 (2017) .

24 Q. An et al. (DAMPE), Science Adv. 5, eaax3793 (2019).

25] P. Brogi and K. Kobayashi (CALET), in PoS (ICRC2021) 101 (2021) .

26] M. Aguilar et al. (AMS02), Phys. Rev. Lett. 115, 211101 (2015).

27 F. Alemanno et al. (DAMPE), Phys. Rev. Lett. 126, 201102 (2021)

28] Y. S. Yoon et al., Astrophys. J. 728, 122 (2011); Astrophys. J. 839, 5 (2017) .

29] O. Adriani et al. (CALET), Phys. Rev. Lett. 125, 251102 (2020) .

30] P. Maestro (CALET), in PoS (ICRC2021) 093 (2021) .

31] J. J. Engelmann et al. (HEAO-3-C2), Astron. Astrophys. 233, 96 (1990) .

32 D. Müller et al., Astrophys. J. 374, 356 (1991).

33] A. Panov et al. (ATIC), Bull. Russian Acad. Sci. 73, 564 (2009).

34 M. Ave et al. (TRACER), Astrophys. J. 678, 262 (2008).

35] M. Aguilar et al. (AMS), Phys. Rev. Lett. 119, 251101 (2017).

36] O. Adriani et al. (PAMELA), Astrophys. J. 93, 791 (2014) .

37 H. S. Ahn et al. (CREAM), Astrophys. J. 707, 593 (2009).

38] E. Atkin et al. (NUCLEON), JCAP 2017, 020 (2017) .

39] M. Simon et al., Astrophys. J. 239, 712 (1980).

40] Y. Akaike and P. Maestro (CALET), in PoS (ICRC2021) 112 (2021).

[41] O. Adriani et al., Astrophys. J. 791, 93 (2014) .

[42] M. Aguilar et al., Phys. Rev. Lett. 120, 021101 (2018).

[43] O. Adriani et al. (CALET), Phys. Rev. Lett. 126, 241101 (2021).

[44] J.J. Engelmann et al., Astron. Astrophys. 96, 233 (1990)

[45] A.D. Panov et al., Proc. of 30th ICRC 2, 3 (2008).

[46] H. Ahn et al., Astropart. Phys. 30, 133 (2008) .

[47] A. Obermeier et al., Astropart. Phys. 752, 69 (2012)

$48]$ V. Grebenyuk et al., Adv. Sp. Res. 64, 2559 (2019)

49 F. Stolzi, C. Checchia and Y. Akaike (CALET), in PoS (ICRC2021) 109 (2021).

50] A. Panov et al. Bull. Russian Acad. Sci. 73 (2009) 564

[51] M. Ave et al. Astrophys. J. 678 (2008) 262-273.

[52] D. Müller et al. Astrophys. J. 374 (1991) 356 .

53] F. Aharonian et al. Phys. Rev. D 75 (2007) 042004

54 V. Grebenyuk et al. Adv. in Space Res. 64 (2019) 2546.

$55]$ M. Ichimura et al. Phys. Rev. D 48 (1993) 1949.

56] M. Aguilar et al. Phys. Rev. Lett. 126 (2021) 041104.

57 H.S. Ahn et al. Astrophys. J. 707 (2009) 593.

58] J.J. Engelmann et al. Astron. Astrophys. 233 (1990) 96

59] EPICS http: / / cosmos.n. kanagawa-u.ac.jp/EPICSHome/.

60] B.F. Rauch et al., ApJ, 697(2009) 2083-2088, arXiv:0906.2021 .

[61] R.P. Murphy et al., ApJ, 831(2016) 2083-2088, arXiv:1608.08183 .

[62] N. Walsh et. al, Advances in Space Research (pending).

63] W.R. Binns, M.H. Israel, M.E. Wiedenbeck, et al., in PoS (ICRC2019) 673 (2019)

[64] W.V. Zober, B.F. Rauch and A.W. Ficklin (CALET), in PoS (ICRC2021) 124 (2021).

[65] A.W. Ficklin, B.F. Rauch and W.V. Zober (CALET), in PoS (ICRC2021) 069 (2021).

[66] N. Walsh, Doctoral dissertation, Washington University in St. Louis (2020) .

[67] B.F. Rauch and W. R. Binns (CALET), in PoS (ICRC2019) 130 (2019) .

[68] S. Miyake (CALET), in PoS (ICRC2021) 1270 (2021).

69 N. Cannady (CALET), in PoS (ICRC2021) 604 (2021).

[70] Y. Kawakubo (CALET), in PoS (ICRC2021) 957 (2021).

[71] M. Mori (CALET), in PoS (ICRC2021) 619 (2021).

[72] A. Bruno et al. (CALET), in PoS (ICRC2021) $1295^{\circ}$ (2021). 


\section{Full Authors List: CALET}

O. Adriani ${ }^{1,2}$, Y. Akaike ${ }^{3,4}$, K. Asano ${ }^{5}$, Y. Asaoka ${ }^{5}$, E. Berti ${ }^{1,2}$, G. Bigongiari ${ }^{6,7}$, W. R. Binns ${ }^{8}$, M. Bongi ${ }^{1,2}$, P. Brogi ${ }^{6,7}$, A. Bruno ${ }^{9,10}$, J. H. Buckley ${ }^{8}$, N. Cannady ${ }^{11,12,13}$, G. Castellini ${ }^{14}$, C. Checchia ${ }^{6}$, M. L. Cherry ${ }^{15}$, G. Collazuol ${ }^{16,17}$, K. Ebisawa ${ }^{18}$, A. W. Ficklin ${ }^{15}$, H. Fuke ${ }^{18}$, S. Gonzi ${ }^{1,2}$, T. G. Guzik ${ }^{15}$, T. Hams ${ }^{11}$, K. Hibino ${ }^{19}$, M. Ichimura ${ }^{20}$, K. Ioka $^{21}$, W. Ishizaki ${ }^{5}$, M. H. Israel ${ }^{8}$, K. Kasahara ${ }^{22}$, J. Kataoka ${ }^{23}$, R. Kataoka ${ }^{24}$, Y. Katayose ${ }^{25}$, C. Kato $^{26}$, N. Kawanaka ${ }^{27,28}$, Y. Kawakubo ${ }^{15}$, K. Kobayashi ${ }^{3,4}$, K. Kohri ${ }^{29}$, H. S. Krawczynski ${ }^{8}$, J. F. Krizmanic ${ }^{11,12,13}$, P. Maestro6,7, P. S. Marrocchesi 6,7, A. M. Messineo ${ }^{30,7}$, J.W. Mitchel112, S. Miyake ${ }^{32}$, A. A. Moiseev ${ }^{33,12,13 \text {, }}$ M. Mori ${ }^{34}$, N. Mori $^{2}$, H. M. Motz ${ }^{35}$, K. Munakata ${ }^{26}$, S. Nakahira ${ }^{18}$, J. Nishimura ${ }^{18}$, G. A. de Nolfo ${ }^{9}$, S. Okuno ${ }^{19}$, J. F. Ormes $^{36}$, N. Ospina ${ }^{16,17}$, S. Ozawa ${ }^{37}$, L. Pacini ${ }^{1,14,2}$, P. Papini ${ }^{2}$, B. F. Rauch ${ }^{8}$, S. B. Ricciarini ${ }^{14,2}$, K. Sakai ${ }^{11,12,13 \text {, }}$ T. Sakamoto ${ }^{38}$, M. Sasaki ${ }^{33,12,13}$, Y. Shimizu ${ }^{19}$, A. Shiomi ${ }^{39}$, P. Spillantini ${ }^{1}$, F. Stolzi ${ }^{6,7}$, S. Sugita ${ }^{38}$, A. Sulaj ${ }^{6,7}$, M. Takita ${ }^{5}$, T. Tamura ${ }^{19}$, T. Terasawa ${ }^{40}$, S. Torii ${ }^{3}$, Y. Tsunesada ${ }^{41}$, Y. Uchihori ${ }^{42}$, E. Vannuccini ${ }^{2}$, J. P. Wefel ${ }^{15}$, K. Yamaoka ${ }^{43}$, S. Yanagita ${ }^{44}$, A. Yoshida ${ }^{38}$, K. Yoshida ${ }^{22}$, and W. V. Zober ${ }^{8}$

${ }^{1}$ Department of Physics, University of Florence, Via Sansone, 1, 50019 Sesto, Fiorentino, Italy, ${ }^{2}$ INFN Sezione di Florence, Via Sansone, 1, 50019 Sesto, Fiorentino, Italy, ${ }^{3}$ Waseda Research Institute for Science and Engineering, Waseda University, 17 Kikuicho, Shinjuku, Tokyo 162-0044, Japan, ${ }^{4}$ JEM Utilization Center, Human Spaceflight Technology Directorate, Japan Aerospace Exploration Agency, 2-1-1 Sengen, Tsukuba, Ibaraki 305-8505, Japan, ${ }^{5}$ Institute for Cosmic Ray Research, The University of Tokyo, 5-1-5 Kashiwa-no-Ha, Kashiwa, Chiba 277-8582, Japan, ${ }^{6}$ Department of Physical Sciences, Earth and Environment, University of Siena, via Roma 56, 53100 Siena, Italy, ${ }^{7}$ INFN Sezione di Pisa, Polo Fibonacci, Largo B. Pontecorvo, 3, 56127 Pisa, Italy, ${ }^{8}$ Department of Physics and McDonnell Center for the Space Sciences, Washington University, One Brookings Drive, St. Louis, Missouri 63130-4899, USA, ${ }^{9}$ Heliospheric Physics Laboratory, NASA/GSFC, Greenbelt, Maryland 20771, USA, ${ }^{10}$ Department of Physics, Catholic University of America, Washington, DC 20064, USA, ${ }^{11}$ Center for Space Sciences and Technology, University of Maryland, Baltimore County, 1000 Hilltop Circle, Baltimore, Maryland 21250, USA, ${ }^{12}$ Astroparticle Physics Laboratory, NASA/GSFC, Greenbelt, Maryland 20771, USA, ${ }^{13}$ Center for Research and Exploration in Space Sciences and Technology, NASA/GSFC, Greenbelt, Maryland 20771, USA, ${ }^{14}$ Institute of Applied Physics (IFAC), National Research Council (CNR), Via Madonna del Piano, 10, 50019 Sesto, Fiorentino, Italy, ${ }^{15}$ Department of Physics and Astronomy, Louisiana State University, 202 Nicholson Hall, Baton Rouge, Louisiana 70803, USA, ${ }^{16}$ Department of Physics and Astronomy, University of Padova, Via Marzolo, 8, 35131 Padova, Italy, ${ }^{17}$ INFN Sezione di Padova, Via Marzolo, 8, 35131 Padova, Italy, ${ }^{18}$ Institute of Space and Astronautical Science, Japan Aerospace Exploration Agency, 3-1-1 Yoshinodai, Chuo, Sagamihara, Kanagawa 252-5210, Japan, ${ }^{19}$ Kanagawa University, 3-27-1 Rokkakubashi, Kanagawa, Yokohama, Kanagawa 221-8686, Japan, ${ }^{20}$ Faculty of Science and Technology, Graduate School of Science and Technology,, Hirosaki University, 3, Bunkyo, Hirosaki, Aomori 036-8561, Japan, ${ }^{21}$ Yukawa Institute for Theoretical Physics, Kyoto University, Kitashirakawa Oiwakecho, Sakyo, Kyoto 606-8502, Japan, ${ }^{22}$ Department of Electronic Information Systems, Shibaura Institute of Technology, 307 Fukasaku, Minuma, Saitama 337-8570, Japan, ${ }^{23}$ School of Advanced Science and Engineering, Waseda University, 3-4-1 Okubo, Shinjuku, Tokyo 169-8555, Japan, ${ }^{24}$ National Institute of Polar Research, 10-3, Midori-cho, Tachikawa, Tokyo 190-8518, Japan, ${ }^{25}$ Faculty of Engineering, Division of Intelligent Systems Engineering, Yokohama National University, 79-5 Tokiwadai, Hodogaya, Yokohama 240-8501, Japan, ${ }^{26}$ Faculty of Science, Shinshu University, 3-1-1 Asahi, Matsumoto, Nagano 390-8621, Japan, ${ }^{27}$ Hakubi Center, Kyoto University, Yoshida Honmachi, Sakyo-ku, Kyoto 606-8501, Japan, ${ }^{28}$ Department of Astronomy, Graduate School of Science, Kyoto University, Kitashirakawa Oiwake-cho, Sakyo-ku, Kyoto 6068502, Japan, ${ }^{29}$ Institute of Particle and Nuclear Studies, High Energy Accelerator Research Organization, 1-1 Oho, Tsukuba, Ibaraki 305-0801, Japan, ${ }^{30}$ University of Pisa, Polo Fibonacci, Largo B. Pontecorvo, 3, 56127 Pisa, Italy, ${ }^{31}$ Astroparticle Physics Laboratory, NASA/GSFC, Greenbelt, Maryland 20771, USA, ${ }^{32}$ Department of Electrical and Electronic Systems Engineering, National Institute of Technology, Ibaraki College, 866 Nakane, Hitachinaka, Ibaraki 312-8508, Japan ${ }^{33}$ Department of Astronomy, University of Maryland, College Park, Maryland 20742, USA, ${ }^{34}$ Department of Physical Sciences, College of Science and Engineering, Ritsumeikan University, Shiga 525-8577, Japan, ${ }^{35}$ Faculty of Science and Engineering, Global Center for Science and Engineering, Waseda University, 3-4-1 Okubo, Shinjuku, Tokyo 169-8555, Japan, ${ }^{36}$ Department of Physics and Astronomy, University of Denver, Physics Building, Room 211, 2112 East Wesley Avenue, Denver, Colorado 80208-6900, USA, ${ }^{37}$ Quantum ICT Advanced Development Center, National Institute of Information and Communications Technology, 4-2-1 Nukui-Kitamachi, Koganei, Tokyo 184-8795, Japan, ${ }^{38}$ College of Science and Engineering, Department of Physics and Mathematics, Aoyama Gakuin University, 5-10-1 Fuchinobe, Chuo, Sagamihara, Kanagawa 252-5258, Japan, ${ }^{39}$ College of Industrial Technology, Nihon University, 1-2-1 Izumi, Narashino, Chiba 275-8575, Japan ${ }^{40}$ RIKEN, 2-1 Hirosawa, Wako, Saitama 351-0198, Japan, ${ }^{41}$ Division of Mathematics and Physics, Graduate School of Science, Osaka City University, 3-3-138 Sugimoto, Sumiyoshi, Osaka 558-8585, Japan, ${ }^{42}$ National Institutes for Quantum and Radiation Science and Technology, 4-9-1 Anagawa, Inage, Chiba 263-8555, Japan, ${ }^{43}$ Nagoya University, Furo, Chikusa, Nagoya 464-8601, Japan, ${ }^{44}$ College of Science, Ibaraki University, 2-1-1 Bunkyo, Mito, Ibaraki 310-8512, Japan 